\title{
Multi-product economic lot scheduling problem with separate production lines for manufacturing and remanufacturing
}

\author{
Ruud Teunter $^{\mathrm{a}, *}$, Konstantinos Kaparis ${ }^{\mathrm{a}, 1}$, Ou Tang ${ }^{\mathrm{b}, 2}$ \\ a Department of Management Science, Lancaster University Management School, Lancaster LA1 4YX, UK \\ ${ }^{\mathrm{b}}$ Department of Production Economics, Linköping Institute of Technology, SE-581 83 Linköping, Sweden
}

Available online 7 August 2007

\begin{abstract}
We study the economic lot scheduling problem with two production sources, manufacturing and remanufacturing, for which operations are performed on separate, dedicated lines. We develop an exact algorithm for finding the optimal common-cycle-time policy. The algorithm combines a search for the optimal cycle time with a mixed integer programming (MIP) formulation of the problem given a fixed cycle time. Using case study data from an auto part producer, we perform a sensitivity study on the effects of key problem parameters such as demand rates and return fractions. Furthermore, by comparing to results in Tang and Teunter [Tang, O., Teunter, R.H., 2006. Economic lot scheduling problem with returns. Production and Operations Management] for the situation where all operations are performed on the same line, we analyze the cost benefits of using dedicated lines.
\end{abstract}

(C) 2007 Elsevier B.V. All rights reserved.

Keywords: ELSP; Returns; Remanufacturing; Reverse logistics

\section{Introduction}

In recent years, closed-loop supply chain management has emerged as a new research area. The driving forces are legislations, potential profit margins of reusing products and customers' awareness of environment-friendly products. Even though the benefit of product recovery is obvious at strategic level, managing return flows is difficult at operational level (Guide, 2000). According to a survey study by Sundin et al. (2005), half of the companies that face return flows report a lack of control in managing them. Therefore, there is a pressing need to develop new models and extend existing models to support decision making in closedloop supply chains.

\footnotetext{
* Corresponding author. Tel.: +44 1524 592384/2214; fax: +44 1524844885.

E-mail addresses: r.teunter@lancaster.ac.uk (R. Teunter), k.kaparis@lancaster.ac.uk (K. Kaparis), ou.tang@ipe.liu.se (O. Tang).

1 Tel.: +44 1524 592384/2214; fax: +44 1524844885 .

2 Tel.: +46 13 281773; fax: +46 13288975 .
} 
This paper deals with the multi-product economic lot scheduling problem with returns (ELSPR), where remanufactured returns are substitutes for newly manufactured products. Tang and Teunter (2006) recently provide the first analysis of the multi-product ELSPR. We refer interested readers to that paper for a discussion on key differences with the traditional ELSP, and to Elmaghraby (1978) for a general discussion of the ELSP. This paper differs from that by Tang and Teunter (2006) in that we assume dedicated production lines for manufacturing and remanufacturing, whereas they assume that all operations are performed on the same line.

The single line assumption in Tang and Teunter (2006) reflects the current operations in a case company that we will name AutoPart, which remanufactures car parts for the service market. Our dedicated lines assumption is motivated by this case study, as AutoPart now considers setting up a separate remanufacturing line.

The question of when or whether to set up dedicated production lines for remanufacturing is relevant in general for firms that both manufacture and remanufacture products. When return rates are low, as they typically will be when products are at the beginning of their life cycle, it may be preferable to perform remanufacturing operations on existing manufacturing lines. However, at later stages of the life cycle, return rates may increase up to levels where it becomes more economical to set up dedicated lines.

A number of authors have studied the ELSP with multiple, either identical or non-identical, production lines. This problem is also referred to as the multi-machine ELSP. Interested readers are referred to Bollapragada and Rao (1999) for a short review. However, to the best of our knowledge, none of these authors have addressed the situation where one product is produced on multiple machines, which is an important characteristic of the ELSPR with dedicated lines. Indeed, as will appear from our analysis, the production of a product on two different lines is the key complicating factor for the ELSPR.

In this paper, we present an algorithm for determining the optimal common-cycle-time policy for the ELSPR with dedicated lines, where backlogs and lost sales are not allowed. The restricted focus on common-cycletime policies mirrors the development of the ELSP literature without returns. A policy with a common cycle time is easier to apply (in fact, AutoPart uses a common cycle time) and easier to analyze as well. We illustrate the algorithm using data from AutoPart and discuss the advantages for that company in switching from a hybrid manufacturing/remanufacturing line to dedicated lines. Furthermore, a sensitivity analysis provides general insights that are useful for companies in deciding when to switch.

The remainder of this paper is structured as follows. In the next section, we describe the system and the ELSPR with dedicated lines mathematically. Moreover, we explain why it is more difficult to analyze than the ELSPR with a single line. In Section 3, we present the algorithm. In Section 4, the algorithm is applied to data from AutoPart and a sensitivity analysis is performed. Furthermore, the results are compared with those for the single line situation. We end in Section 5 with conclusions and suggestions for future studies.

\section{System description and problem formulation}

We study a production system where several products (models) are produced by either manufacturing or by remanufacturing of returned cores. Since the quality of manufactured and remanufactured products is the same, the finished (re)manufactured products will enter into a joint serviceable stock. Manufacturing and remanufacturing have their own dedicated production lines. As is common in the ELSP literature, we assume constant demand and manufacturing rates, constant return and remanufacturing rates as well as sequence independent setup costs/times. These assumptions are justified for AutoPart. The demand and return rates are large (more than 100,000 water pumps are demanded and more than 20,000 are returned per year) and stable. Each (re)manufactured pump undergoes the same (re)manufacturing steps, and (re)manufacturing times show little variability as a result. The major setup activities are a call for new materials and a change of the workbench (layout), and these are sequence independent.

As mentioned before, we restrict our attention to policies with a common cycle time for all products (as is currently used by AutoPart) and for both lines. Fig. 1 illustrates the inventory patterns for such a policy for an arbitrary example (the blocks with stripes indicate idle time). Note that the minimum returns and serviceables inventory are always set to zero in order to minimize holding costs. Also note that the production schedule in Fig. 1 is clearly suboptimal. Manufacturing of product 2, for instance, can be delayed because the line is idle 


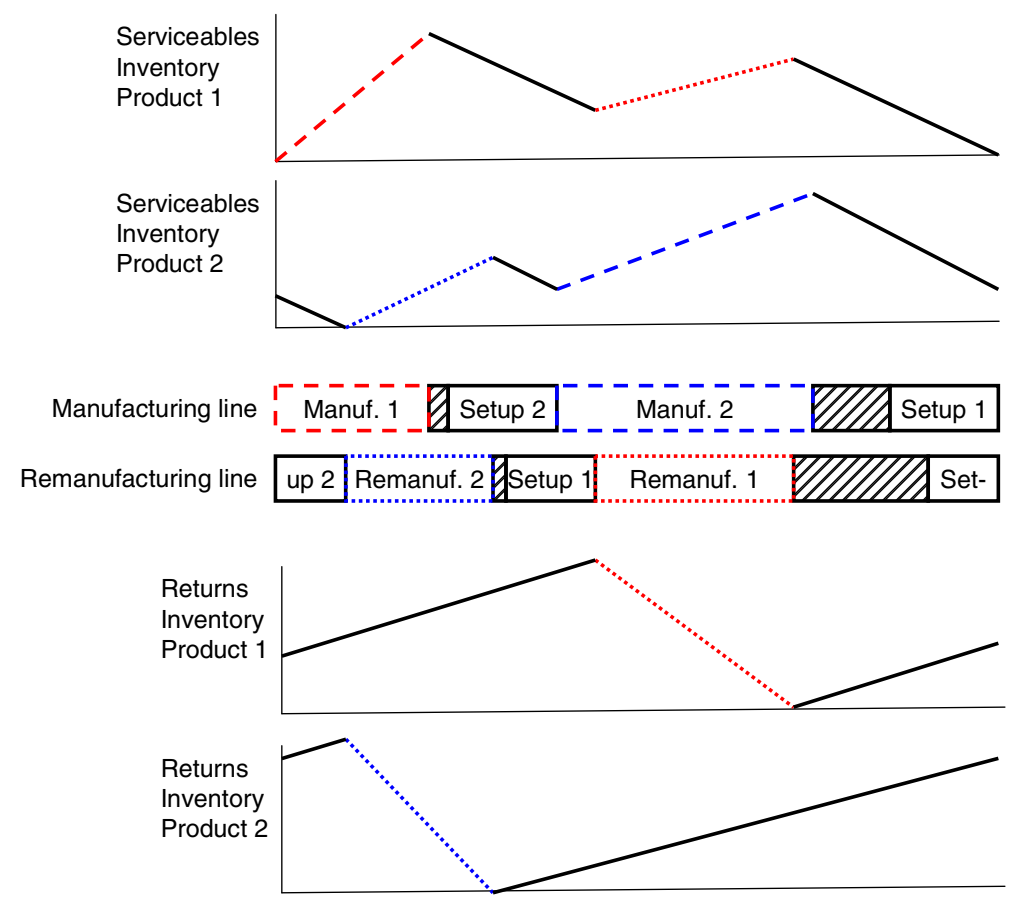

Fig. 1. Example of inventory patterns during a cycle with two products. The blocks with stripes indicate idle time.

for some time afterwards, and doing so would reduce the serviceable inventory level product 2 (and not affect the returns inventory level).

The objective is to find the optimal policy: that is, to find the cycle time and the production start times for (re)manufacturing lots that minimize the cost per time unit. Included are the setup costs for manufacturing and remanufacturing, the holding cost (per product per time unit) for returned products, and the holding cost for serviceable products. Note that variable production costs are not included. Since disposal is not considered and all returned cores can be and are remanufactured, the fractions of demand satisfied by manufactured and remanufactured products are fixed, and therefore the variable production costs are not affected (in the long run).

We do not assume that both the manufacturing rate and the remanufacturing rate have to be at least as large as the demand rate. Especially the assumption that the remanufacturing rate needs to be at least as large as the demand rate, can be unrealistic. In many real-life cases, including the case of Autopart as we will see, return rates are below 30\% and hence the relatively small remanufacturing line does not (have to) produce at the overall demand rate. We do assume that the maximum of the manufacturing rate and the remanufacturing rate is at least as large as the demand rate. This assumption is satisfied for the case of AutoPart (see Section 4). Furthermore, in general, the assumption will hold unless the major line cannot satisfy all demand for just one particular product, even if it is fully dedicated to that product, which seems unrealistic. We remark that without this assumption, the analysis would be more complex and tedious, as we will indicate in Section 4.

There are two main reasons why the ELSPR with dedicated lines is more difficult to analyze than the ELSPR with a single line. First, manufacturing and remanufacturing intervals for a certain product may overlap. Second, since the demand rate can be larger than the (re)manufacturing rate, serviceables inventory can decrease during production. Both complications lead to more complex serviceable inventory patterns, as we will see in the next section.

The following notation will be used.

$N \quad$ number of products

$k_{i} \quad$ class to which product $i$ belongs (classes are defined in Section 3)

$D_{i} \quad$ constant demand rate for product $i=1,2, \ldots, N$, units/time unit

$h_{i}^{s}\left(h_{i}^{r}\right)$ inventory holding cost for serviceable (recoverable) inventory, $€ /$ unit/time unit 
$K_{i}^{m}\left(K_{i}^{r}\right)$ setup costs for manufacturing (remanufacturing), $€ / l o t$

$P_{i}^{m}\left(P_{i}^{r}\right)$ manufacturing (remanufacturing) rate, units/time unit

$s_{i}^{m}\left(s_{i}^{r}\right)$ setup time of manufacturing (remanufacturing), time units

$T \quad$ common cycle time, time units

$x_{i}^{m}\left(x_{i}^{r}\right)$ time at which the manufacturing (remanufacturing) of product $i$ starts, time units

$\beta_{i} \quad$ constant remanufacturable return proportion of product $i, 0 \leqslant \beta_{i}<1$

$\rho_{i}^{m} \quad=\left(1-\beta_{i}\right) D_{i} / P_{i}^{m}$

$\rho_{i}^{r} \quad=\beta_{i} D_{i} / P_{i}^{r}$

$f(z) \quad= \begin{cases}z & \text { if } z \geqslant 0 \\ T+z & \text { if } z<0\end{cases}$

$[z]^{+} \quad=\max \{z, 0\}$

$[z]^{-}=\max \{-z, 0\}$

\section{The algorithm}

The algorithm combines a search for the optimal cycle time with a mixed integer programming (MIP) formulation of the problem given a fixed cycle time. The search procedure is straightforward and shortly discussed at the end of this section. Next, we will derive the MIP formulation. The derivation is similar to that by Tang and Teunter (2006) for the single line case, but more complex since we are dealing with two lines and furthermore have relaxed their assumption that both production rates are larger than the demand rate.

Essential for the derivation of the algorithm are three results that we will present in the form of three lemmas. The first lemma gives the smallest feasible cycle time. Obviously, only cycle times of at least this value need to be considered in the search for the optimal cycle time. The second and third lemma show that, for a given cycle time, the cost per time unit can be linearized, which leads to the MIP formulation.

Lemma 1. For a fixed cycle timeT, a feasible solution exists if and only if $T \geqslant T_{\min }=\max \left\{T_{\min }^{m}, T_{\min }^{r}\right\}$, where

$$
T_{\min }^{m}=\frac{\sum_{i=1}^{N} s_{i}^{m}}{1-\sum_{i=1}^{N} \rho_{i}^{m}} \quad \text { and } \quad T_{\min }^{r}=\frac{\sum_{i=1}^{N} s_{i}^{r}}{1-\sum_{i=1}^{N} \rho_{i}^{r}} .
$$

Proof. This is a straightforward application from the minimum required cycle time formula for the traditional ELSP without returns. See e.g. Axsäter (2006).

Lemmas 2 and 3 are on the cost associated with a certain inventory policy. The relevant cost is the cost per time unit, averaged over any interval of duration $T$. For ease of notation, we will simply use the cost per time unit in the rest of this paper. Both lemmas distinguish three classes of products, defined below, which differ in the ideal serviceable inventory pattern (with the lowest average cost per time unit). Lemma 2 gives the cost associated with the ideal pattern for each class. Lemma 3 gives the additional cost for each class.

The descriptions of the three classes and the corresponding ideal inventory patterns (that are depicted graphically in Appendix A) are as follows:

- Class 1: $P_{i}^{m}>D_{i}$ and $P_{i}^{r}>D_{i}$. Inventory increases if either line produces.

- Class 2: $P_{i}^{m}>D_{i}$ and $P_{i}^{r} \leqslant D_{i}$. Inventory increases during manufacturing, but not during remanufacturing.

- Class 3: $P_{i}^{m} \leqslant D_{i}$ and $P_{i}^{r}>D_{i}$. Inventory increases during remanufacturing, but not during manufacturing.

Recall from Section 2 that we assume the largest of the two production rates to be more than the demand rate, so we do not consider an additional class with both production rates equal to or smaller than the demand rate. As argued in Section 1, this assumption is realistic and avoids the analysis of this class (which turns out to be more complex than that for the other classes). 
Lemma 2. The minimum cost per time unit for product $i$ is

$$
I C_{i}=\frac{K_{i}^{m}+K_{i}^{r}}{T}+\frac{1}{2} h_{i}^{r} T D_{i} \beta_{i}\left(1-\rho_{i}^{r}\right)+\frac{1}{2} h_{i}^{s} T F_{i}\left(k_{i}\right),
$$

where factor $F_{i}(k)$ is defined as follows:

\begin{tabular}{ll}
\hline$k$ & $F_{i}(k)$ \\
\hline 1 & $\beta_{i} \rho_{i}^{r}\left(P_{i}^{r}-D_{i}\right)+\left(1-\beta_{i}\right) \rho_{i}^{m}\left(P_{i}^{m}-D_{i}\right)$ \\
2 & $\rho_{i}^{r}\left(1-\rho_{i}^{m}\right)\left(D_{i}-P_{i}^{r}\right)+\rho_{i}^{m}\left(1-\rho_{i}^{r}\right)\left(P_{i}^{m}-D_{i}\right)$ \\
3 & $\rho_{i}^{r}\left(1-\rho_{i}^{m}\right)\left(P_{i}^{r}-D_{i}\right)+\rho_{i}^{m}\left(1-\rho_{i}^{r}\right)\left(D_{i}-P_{i}^{m}\right)$ \\
\hline
\end{tabular}

Proof. See Appendix A.

Lemma 3. The additional cost (on top of the minimum cost given by Lemma 2) per time unit for a product $i$ is

$$
A C_{i}=h_{i}^{s}\left(e_{i}\left(k_{i}\right)\left[f\left(x_{i}^{r}-x_{i}^{m}\right)-T d_{i}\left(k_{i}\right)\right]^{-}+l_{i}\left(k_{i}\right)\left[f\left(x_{i}^{r}-x_{i}^{m}\right)-T d_{i}\left(k_{i}\right)\right]^{+}\right),
$$

where factors $d_{i}(k), e_{i}(k)$ and $l_{i}(k)$ are defined as follows:

\begin{tabular}{llll}
\hline$k$ & $d_{i}(k)$ & $e_{i}(k)$ & $l_{i}(k)$ \\
\hline 1 & $1-\beta_{i}$ & $P_{i}^{r} \rho_{i}^{r}$ & $P_{i}^{m} \rho_{i}^{m}$ \\
2 & $1-\rho_{i}^{r}$ & $P_{i}^{r} \rho_{i}^{r}$ & $P_{i}^{r}\left(1-\rho_{i}^{r}\right)$ \\
3 & $1-\rho_{i}^{m}$ & $P_{i}^{m}\left(1-\rho_{i}^{m}\right)$ & $P_{i}^{m} \rho_{i}^{m}$ \\
\hline
\end{tabular}

Proof. See Appendix B.

Using Lemmas 1-3 and applying some standard linearization techniques (see e.g. Winston, 1991) using a large number, $M$, the ELSP with returns can be formulated as the following mathematical programming problem. Note that $x, y, U, V$ and $Z$ (with indices in subscript and indicators in superscript) are all decision variables in this MIP. However, the times $x$ when (re)manufacturing batches start are the only 'real' variables. The $U, V$ and $Z$ variables are only needed, together with $(2 \mathrm{a}-\mathrm{c})$, to complete the cost linearization by getting rid of the []$^{-}$and []$^{+}$operators. The binary $y^{\mathrm{T}}$ variables are only needed to 'correct' a negative duration between manufacturing and remanufacturing in case remanufacturing starts first (which is done by adding $\mathrm{T}$ in such a case). The binary $y^{m}$ and $y^{r}$ variables are help-variables in ensuring that no two manufacturing periods overlap (3ab), no two remanufacturing periods overlap ( $3 \mathrm{~cd})$, and all setup and production periods fall within the cycle interval that without loss of generalization is assumed to start at time 0 and end at time $T(4 a-d)$.

$$
\underset{U_{i}, V_{i}}{\operatorname{Min}} \sum_{i=1}^{N}\left(I C_{i}+h_{i}^{s} D_{i}\left(e_{i}(k) U_{i}+l_{i}(k) V_{i}\right)\right)
$$

such that

$U_{i} \geqslant Z_{i}$ for all $i \in\{1, \ldots, N\}$,

$U_{i} \geqslant 0$ for all $i \in\{1, \ldots, N\}$,

$V_{i} \geqslant-Z_{i}$ for all $i \in\{1, \ldots, N\}$,

$V_{i} \geqslant 0$ for all $i \in\{1, \ldots, N\}$,

$Z_{i}=x_{i}^{r}-x_{i}^{m}-T d_{i}(k)+T y_{i}^{\mathrm{T}}$ for all $i \in\{1, \ldots, N\}$,

$x_{i}^{r}-x_{i}^{m}+M y_{i}^{\mathrm{T}} \geqslant 0$ for all $i \in\{1, \ldots, N\}$,

$-\left(x_{i}^{r}-x_{i}^{m}\right)+M\left(1-y_{i}^{\mathrm{T}}\right) \geqslant 0$ for all $i \in\{1, \ldots, N\}$, 


$$
\begin{aligned}
& x_{i}^{m}+T \rho_{i}^{m}-x_{j}^{m}+s_{j}^{m} \leqslant M y_{i, j}^{m} \quad \text { for all } i, j \in\{1, \ldots, N\}: j>i, \\
& x_{j}^{m}+T \rho_{i}^{m}-x_{i}^{m}+s_{i}^{m} \leqslant M\left(1-y_{i, j}^{m}\right) \text { for all } i, j \in\{1, \ldots, N\}: j>i, \\
& x_{i}^{r}+T \rho_{i}^{r}-x_{j}^{r}+s_{j}^{r} \leqslant M y_{i, j}^{r} \quad \text { for all } i, j \in\{1, \ldots, N\}: j>i, \\
& x_{j}^{r}+T \rho_{i}^{r}-x_{i}^{r}+s_{i}^{r} \leqslant M\left(1-y_{i, j}^{r}\right) \text { for all } i, j \in\{1, \ldots, N\}: j>i, \\
& x_{i}^{m} \geqslant s_{i}^{m} \text { for all } i \in\{1, \ldots, N\}, \\
& x_{i}^{r} \geqslant s_{i}^{r} \text { for all } i \in\{1, \ldots, N\}, \\
& x_{i}^{m}+T \rho_{i}^{m} \leqslant T \text { for all } i \in\{1, \ldots, N\}, \\
& x_{i}^{r}+T \rho_{i}^{r} \leqslant T \text { for all } i \in\{1, \ldots, N\}, \\
& T \geqslant T_{\min }, \\
& U_{i}, V_{i}, x_{i}^{m}, x_{i}^{r}, T \text { Continuous variables, } \\
& y_{i}^{\mathrm{T}}, y_{i, j}^{m}(j>i), y_{i, j}^{r}(j>i) \quad \text { Binary }(0 \text { or } 1) \text { variables, } \\
& M \quad \text { Large number. }
\end{aligned}
$$

In total (inserting (2a) into (1a) and (1c)), this formulation has $4 N+1$ continuous variables, $N^{2}$ binary variables and $2 N^{2}+8 N+1$ inequalities.

Note that for a fixed value of the cycle time $T, I C_{i}$ is a constant and hence the mathematical program is a mixed integer linear program (MIP) that can be solved by standard software packages such as CPLEX.

By combining the derived MIP for a given cycle time with a search for the cycle time, the optimal solution is determined. Obviously, only feasible cycle times $T \geqslant T_{\min }$ need to be considered. In the numerical experiments described in the next section, we used complete search with a small (1 hour) grid between $T_{\min }$ and a sufficiently large maximum value (at least twice the optimal cycle time). Our numerical results show that the cost of the optimal solution is unimodal in the cycle time for all considered examples, which suggests that faster search procedures can actually be applied (although we have no formal proof of unimodality in general).

\section{Numerical examples}

Recall from Section 1 that this research was mainly motivated by a case company (AutoPart) that currently uses a single line for both manufacturing and remanufacturing operations, but is considering a change to separate lines. This change involves the allocation of machines and workers over the two lines. Before describing this allocation, we first need to provide some production line details. A (combined or dedicated) line consists of four workstations: pressing, assembly, quality control and painting. Each workstation consists of multiple identical machines and workers. Pressing machines are currently the production bottleneck. We remark that, although there are multiple machines in a workstation, they have to work on the same product to coordinate operations with other workstations.

According with the described production line setting, a change from a single to dedicated lines involves the allocation of machines and workers over the dedicated lines. This is done in proportion to the fractions of time spent on (re)manufacturing operations. For instance, if the manufacturing and remanufacturing rates are equal and the return rate is $20 \%$ for all products, then $80 \%$ of machines and workers are allocated to the manufacturing line and $20 \%$ to the remanufacturing line. To continue this example, if the single line production rate is 80 for each product, then the dedicated manufacturing and remanufacturing lines have production rates $0.80 \times 80=64$ and $0.20 \times 80=16$, respectively.

The settings of the above example are, in fact, those for the 'base case' with estimates for one of the key product lines of AutoPart. Other details will be provided in Section 4.1. Besides analyzing this base case, we will perform a sensitivity study in Section 4.2, where the model parameters are modified one at a time. We remark that to be able to assess the effects of changing from a single line to dedicated lines, we analyze the same cases that Tang and Teunter (2006) considered for the single line situation. We also remark that, 
since our interest is purely in the cost per time unit and not in the cost per cycle, we will simply refer to (ideal, additional or total) "cost" instead of "cost per time unit".

\subsection{Base case}

Table 1 gives the values of the model parameters for the base case. See Tang and Teunter (2006) for a discussion. Note that product (pump) number 4 falls into Class 2 and that the other four products fall into Class 1 for this base case.

It turns out that the optimal cycle time is 59 hours. The additional cost is zero for all products and hence the schedule is ideal. So, the total cost is equal to the ideal cost, which is $6.802 € / \mathrm{h}$.

Table 1

Model parameter for the base case (time unit 1 hour, cost unit $1 €$ )

\begin{tabular}{|c|c|c|c|c|c|c|c|c|c|c|c|}
\hline \multirow{2}{*}{$\begin{array}{l}\text { Pump } \\
\text { no. } i\end{array}$} & \multirow{2}{*}{$\begin{array}{l}\text { Pump } \\
\text { type }\end{array}$} & \multirow{2}{*}{$\begin{array}{l}\text { Demand } \\
\text { rate }\end{array}$} & \multicolumn{3}{|c|}{ Manufacturing } & \multicolumn{3}{|c|}{ Remanufacturing } & \multicolumn{2}{|c|}{ Holding cost } & \multirow{2}{*}{$\frac{\begin{array}{l}\text { Return } \\
\text { proportion }\end{array}}{\beta_{i}}$} \\
\hline & & & $\begin{array}{l}\text { Setup } \\
\text { cost } K_{i}^{m}\end{array}$ & $\begin{array}{l}\text { Setup } \\
\text { time } s_{i}^{m}\end{array}$ & $\begin{array}{l}\text { Manu. } \\
\text { rate } P_{i}^{m}\end{array}$ & $\begin{array}{l}\text { Setup } \\
\text { cost } K_{i}^{r}\end{array}$ & $\begin{array}{l}\text { Setup } \\
\text { time } s_{i}^{r}\end{array}$ & $\begin{array}{l}\text { Reman. } \\
\text { rate } P_{i}^{r}\end{array}$ & $\begin{array}{l}\text { Serv. } \\
\text { Inven. } h_{i}^{s}\end{array}$ & $\begin{array}{l}\text { Recov. } \\
\text { Inven. } h_{i}^{r}\end{array}$ & \\
\hline 1 & DL 6 & 9 & 20 & 0.25 & 64 & 20 & 0.25 & 16 & 0.00175 & 0.00088 & 0.2 \\
\hline 2 & DL 7 & 9 & 20 & 0.25 & 64 & 20 & 0.25 & 16 & 0.00263 & 0.00132 & 0.2 \\
\hline 3 & DL 10 & 9 & 20 & 0.25 & 64 & 20 & 0.25 & 16 & 0.00350 & 0.00175 & 0.2 \\
\hline 4 & DL 12 & 30 & 20 & 0.25 & 64 & 20 & 0.25 & 16 & 0.00438 & 0.00219 & 0.2 \\
\hline 5 & DL 16 & 3 & 20 & 0.25 & 64 & 20 & 0.25 & 16 & 0.00525 & 0.00263 & 0.2 \\
\hline
\end{tabular}

Table 2a

Sensitivity results on switching from a single line to separate lines (one parameter is modified from the base case)

\begin{tabular}{|c|c|c|c|c|c|c|c|c|c|}
\hline \multirow{2}{*}{$\begin{array}{l}\text { Modified parameter } \\
\text { (value) }\end{array}$} & \multicolumn{4}{|c|}{ Separate lines } & \multicolumn{4}{|c|}{ Single line } & \multirow{2}{*}{$\begin{array}{l}\text { Cost reduction } \\
(\%)\end{array}$} \\
\hline & $\begin{array}{l}\text { Cycle } \\
\text { time }\end{array}$ & $\begin{array}{l}\text { Cost } \\
\text { ideal }\end{array}$ & $\begin{array}{l}\text { Cost } \\
\text { additional }\end{array}$ & $\begin{array}{l}\text { Cost } \\
\text { total }\end{array}$ & $\begin{array}{l}\text { Cycle } \\
\text { time }\end{array}$ & $\begin{array}{l}\text { Cost } \\
\text { ideal }\end{array}$ & $\begin{array}{l}\text { Cost } \\
\text { additional }\end{array}$ & $\begin{array}{l}\text { Cost } \\
\text { total }\end{array}$ & \\
\hline \multicolumn{10}{|l|}{$\beta$} \\
\hline 0.1 & 54 & 7.386 & 0 & 7.386 & 53 & 7.537 & 0.002 & 7.539 & 2.03 \\
\hline 0.2 & 59 & 6.802 & 0 & 6.802 & 56 & 7.200 & 0.001 & 7.201 & 5.54 \\
\hline 0.3 & 64 & 6.281 & 0 & 6.281 & 57 & 7.016 & 0.006 & 7.022 & 10.55 \\
\hline 0.4 & 68 & 5.921 & 0 & 5.921 & 57 & 6.996 & 0 & 6.996 & 15.37 \\
\hline 0.5 & 66 & 6.043 & 0 & 6.043 & 54 & 7.145 & 0 & 7.145 & 15.42 \\
\hline \multicolumn{10}{|l|}{$D$ (change) } \\
\hline$-30 \%$ & 69 & 5.813 & 0 & 5.813 & 65 & 6.109 & 0 & 6.109 & 4.85 \\
\hline$-15 \%$ & 62 & 6.448 & 0 & 6.448 & 59 & 6.792 & 0 & 6.792 & 5.06 \\
\hline$+0 \%$ & 59 & 6.802 & 0 & 6.802 & 56 & 7.200 & 0.001 & 7.201 & 5.54 \\
\hline$+15 \%$ & 56 & 7.084 & 0 & 7.084 & 53 & 7.539 & 0.040 & 7.579 & 6.53 \\
\hline$+30 \%$ & 55 & 7.301 & 0.037 & 7.338 & 100 & 9.635 & 0.350 & 9.985 & 26.88 \\
\hline \multicolumn{10}{|l|}{$h^{r} / h^{s}$} \\
\hline 0.1 & 62 & 6.414 & 0 & 6.414 & 60 & 6.726 & 0 & 6.726 & 4.64 \\
\hline 0.3 & 61 & 6.611 & 0 & 6.611 & 57 & 6.967 & 0.0001 & 6.967 & 5.11 \\
\hline 0.5 & 59 & 6.802 & 0 & 6.802 & 56 & 7.200 & 0.001 & 7.201 & 5.54 \\
\hline 0.7 & 57 & 6.988 & 0 & 6.988 & 54 & 7.426 & 0.0002 & 7.426 & 5.90 \\
\hline 0.9 & 56 & 7.169 & 0 & 7.169 & 52 & 7.646 & 0.0003 & 7.646 & 6.24 \\
\hline \multicolumn{10}{|l|}{$h^{s}$ (change) } \\
\hline$-50 \%$ & 83 & 4.810 & 0 & 4.810 & 79 & 5.091 & 0 & 5.091 & 5.52 \\
\hline$-25 \%$ & 68 & 5.891 & 0 & 5.891 & 64 & 6.235 & 0 & 6.235 & 5.52 \\
\hline$+0 \%$ & 59 & 6.802 & 0 & 6.802 & 56 & 7.200 & 0.001 & 7.201 & 5.54 \\
\hline$+25 \%$ & 53 & 7.605 & 0 & 7.605 & 50 & 8.050 & 0.005 & 8.055 & 5.59 \\
\hline$+50 \%$ & 48 & 8.331 & 0 & 8.331 & 45 & 8.819 & 0.0009 & 8.819 & 5.53 \\
\hline
\end{tabular}


Table $2 b$

Sensitivity results on switching from a single line to separate lines (one parameter is modified from the base case)

\begin{tabular}{|c|c|c|c|c|c|c|c|c|c|}
\hline \multirow{2}{*}{$\begin{array}{l}\text { Modified parameter } \\
\text { (value) }\end{array}$} & \multicolumn{4}{|c|}{ Separate lines } & \multicolumn{4}{|c|}{ Single line } & \multirow{2}{*}{$\begin{array}{l}\text { Cost reduction } \\
(\%)\end{array}$} \\
\hline & $\begin{array}{l}\text { Cycle } \\
\text { time }\end{array}$ & $\begin{array}{l}\text { Cost } \\
\text { ideal }\end{array}$ & $\begin{array}{l}\text { Cost } \\
\text { additional }\end{array}$ & $\begin{array}{l}\text { Cost } \\
\text { total }\end{array}$ & $\begin{array}{l}\text { Cycle } \\
\text { time }\end{array}$ & $\begin{array}{l}\text { Cost } \\
\text { ideal }\end{array}$ & $\begin{array}{l}\text { Cost } \\
\text { additional }\end{array}$ & $\begin{array}{l}\text { Cost } \\
\text { total }\end{array}$ & \\
\hline \multicolumn{10}{|l|}{$K^{m}$} \\
\hline 100 & 54 & 5.901 & 0 & 5.901 & 48 & 6.23564 & 0.004 & 6.235 & 5.36 \\
\hline 150 & 55 & 6.363 & 0 & 6.363 & 52 & 6.7355 & 0.003 & 6.735 & 5.52 \\
\hline 200 & 59 & 6.802 & 0 & 6.802 & 56 & 7.200 & 0.001 & 7.201 & 5.54 \\
\hline 250 & 62 & 7.215 & 0 & 7.215 & 59 & 7.647 & 0.0004 & 7.647 & 5.65 \\
\hline 300 & 66 & 7.605 & 0 & 7.605 & 62 & 8.050 & 0 & 8.050 & 5.53 \\
\hline \multicolumn{10}{|l|}{$K^{r}$} \\
\hline 100 & 51 & 5.891 & 0 & 5.891 & 48 & 6.236 & 0.0005 & 6.236 & 5.53 \\
\hline 150 & 55 & 6.363 & 0 & 6.363 & 52 & 6.736 & 0.0003 & 6.736 & 5.54 \\
\hline 200 & 59 & 6.802 & 0 & 6.802 & 56 & 7.200 & 0.001 & 7.201 & 5.54 \\
\hline 250 & 62 & 7.215 & 0 & 7.215 & 59 & 7.637 & 0.00004 & 7.637 & 5.53 \\
\hline 300 & 66 & 7.605 & 0 & 7.605 & 62 & 8.051 & 0 & 8.051 & 5.54 \\
\hline \multicolumn{10}{|l|}{$P^{m}$ (single line) } \\
\hline 60 & 62 & 6.449 & 0.032 & 6.481 & 58 & 6.838 & 0.126 & 6.964 & 7.40 \\
\hline 70 & 60 & 6.654 & 0 & 6.654 & 57 & 7.048 & 0.0226 & 7.070 & 5.88 \\
\hline 80 & 59 & 6.802 & 0 & 6.802 & 56 & 7.200 & 0.001 & 7.201 & 5.54 \\
\hline 90 & 58 & 6.916 & 0 & 6.916 & 55 & 7.317 & 0 & 7.317 & 5.48 \\
\hline 100 & 57 & 7.006 & 0 & 7.006 & 54 & 7.409 & 0 & 7.409 & 5.44 \\
\hline \multicolumn{10}{|l|}{$P^{r}$ (single line) } \\
\hline 60 & 60 & 6.716 & 0 & 6.716 & 56 & 7.179 & 0.006 & 7.185 & 6.53 \\
\hline 70 & 59 & 6.765 & 0 & 6.765 & 57 & 7.193 & 0.003 & 7.196 & 5.99 \\
\hline 80 & 59 & 6.802 & 0 & 6.802 & 56 & 7.200 & 0.001 & 7.201 & 5.54 \\
\hline 90 & 59 & 6.831 & 0 & 6.831 & 55 & 7.208 & 0 & 7.208 & 5.23 \\
\hline 100 & 58 & 6.854 & 0 & 6.856 & 55 & 7.214 & 0 & 7.214 & 4.99 \\
\hline \multicolumn{10}{|l|}{$s^{m}$} \\
\hline 0.05 & 59 & 6.802 & 0 & 6.802 & 56 & 7.201 & 0 & 7.201 & 5.54 \\
\hline 0.15 & 59 & 6.802 & 0 & 6.802 & 56 & 7.201 & 0 & 7.201 & 5.54 \\
\hline 0.25 & 59 & 6.802 & 0 & 6.802 & 56 & 7.201 & 0.001 & 7.201 & 5.54 \\
\hline 0.35 & 59 & 6.802 & 0 & 6.802 & 56 & 7.201 & 0.0008 & 7.202 & 5.55 \\
\hline 0.45 & 59 & 6.802 & 0 & 6.802 & 56 & 7.200 & 0.0014 & 7.202 & 5.55 \\
\hline \multicolumn{10}{|l|}{$s^{r}$} \\
\hline 0.05 & 59 & 6.802 & 0 & 6.802 & 56 & 7.201 & 0 & 7.201 & 5.54 \\
\hline 0.15 & 59 & 6.802 & 0 & 6.802 & 56 & 7.201 & 0 & 7.201 & 5.54 \\
\hline 0.25 & 59 & 6.802 & 0 & 6.802 & 56 & 7.201 & 0.001 & 7.201 & 5.54 \\
\hline 0.35 & 59 & 6.802 & 0 & 6.802 & 56 & 7.201 & 0.0005 & 7.201 & 5.54 \\
\hline 0.45 & 59 & 6.802 & 0 & 6.802 & 56 & 7.201 & 0.0008 & 7.202 & 5.55 \\
\hline
\end{tabular}

The optimal single line solution (Tang and Teunter, 2006) had a cycle time of 56 hours, an ideal cost of $7.200 € / \mathrm{h}$ and an additional cost of $0.001 € / \mathrm{h}$. Although this additional cost is very small, its elimination (or, in general, reduction) when separate lines are used will turn out to be a consistent result in the sensitivity study in Section 4.2. We can identify two reasons for the reduction in additional cost from a single to dedicated lines. First, the larger cycle time (from 56 to 59 hours) implies a larger idle time percentage. Since idle time can be inserted before any batch, more idle time makes it easier to start (re)manufacturing batches at (nearly) ideal times. The second reason is that starting times of batches are less restricted when separate lines are used, because manufacturing intervals and remanufacturing intervals are allowed to overlap. So, both effects lead to increased scheduling flexibility, resulting in reduced additional costs.

There is a considerable reduction of $5.5 \%$ in the ideal and total cost. This is related to the lower production rates for the situation with separate lines compared to that with a combined line. The lower production rates result from allocating the resources of the combined line over the two dedicated lines. Lower production rates 
lead to a slower build-up of inventory and therefore to lower inventory costs (for a fixed cycle time). Indirectly, this also explains the larger optimal cycle time by shifting the balance between inventory and setup costs.

\subsection{Sensitivity analysis}

We modify one model parameter at a time from the base case, and do so for all products. For instance, the return rate for each product is 0.4 instead of 0.2 , or $30 \%$ is added to the demand rate for each product. The results are presented in Tables $2 \mathrm{a}$ and $\mathrm{b}$. The last column gives the percentage reduction in total cost for switching from a single line to separate lines.

The first important observation is that switching from a single to separate lines leads to a considerable cost reduction that varies from $2.2 \%$ to $26.5 \%$ and is $6.5 \%$ on average. As expected, the reduction is especially sensitive with respect to the return rate. Performing remanufacturing operations on an 'existing' manufacturing line becomes less efficient as the return rate increases. The reduction is fairly stable with respect to the other parameters with one notable exception. This exceptional case occurs when demand is increased by $30 \%$, and is in fact the only case for which capacity utilization (97.5\%) is so high that the minimum cycle time restriction (see Lemma 1) becomes binding for the single line situation (where it is 100), but not for the separate line situation (where it is 50). This results in a much larger cost reduction for this case.

For most of the cases considered, the additional cost was zero or negligible to start with for the single line situation. Hence, the reduction in mean (i.e. over all cases) total cost is largely due to the reduction in the mean ideal cost. This reduction can be attributed to the smaller production rates after allocating the work force and machinery over the two lines, which results in slower build-up of serviceable stock during production. This also explains why the optimal cycle time increases when switching to separate lines, with the same exceptional case as discussed before.

The cases with high capacity utilization do show that switching to separate lines consistently reduces the additional cost. The additional cost is reduced by more than $70 \%$ for the two cases where it was relatively large (more than $1 \%$ of the total cost) with a single line, and is eliminated for all other cases. As discussed in Section 4.1 , the reduction in additional cost is explained by the increased scheduling flexibility that results from the larger idle time percentage (for the larger cycle time) and from the allowed overlap of manufacturing and remanufacturing intervals.

\section{Conclusions}

We considered the economic lot scheduling problem with separate lines for manufacturing and remanufacturing, and focused on common-cycle-time policies, i.e. policies with the same cycle time for each product and each line. We derived a mixed integer programming (MIP) of the problem for a fixed cycle time, which can be combined with a cycle time search to find an optimal solution. The derivation of the MIP turned out to be more complex than for the situation with a single 'hybrid' line that was analyzed by Tang and Teunter (2006) because of two complication factors: (i) the assumption that all production rates are larger than the demand rates is no longer justified and (ii) manufacturing and remanufacturing intervals may overlap. Both factors lead to more complex serviceable inventory patterns.

In a numerical experiment, we determined the optimal common cycle policy for a base case with estimates for a car-part producer (AutoPart) and modifications from it. The same set of cases was considered for the single line situation by Tang and Teunter (2006), which allowed us to analyze the effects of switching from a single to separate lines. AutoPart is currently considering such a switch, and companies generally do with growing remanufacturing activities.

The numerical experiment revealed that setting up dedicated production lines for manufacturing and remanufacturing can lead to significant reductions in holding costs through lower production rates and increased scheduling flexibility. The average reduction in total cost per time unit was $6.5 \%$. The reduction is sharply increasing in the return rate (to more than $15 \%$ for return rates of $40 \%$ and higher), confirming the intuition that the need for performing remanufacturing operations on a separate line increases with the size of those operations. 
Of course, one should keep in mind that setting up a separate line will create an investment cost that should be traded off against the reduction in operational costs. This trade-off was outside the scope of this paper, but certainly offers an interesting direction for further research. Another direction is to analyze more complex policies without a common cycle times for the two lines or even for products on the same line. Such policies will, however, be more complex to analyze. Finally, heuristic solution methods can be developed for either the common cycle time or more complex policies.

\section{Appendix A. Proof of Lemma 2 (minimum cost per time unit)}

The expression for the setup cost in Lemma 2 results since each product is setup once for manufacturing and once for remanufacturing during each cycle of duration $T$. The inventory of a returned product decreases during its remanufacturing intervals and increases otherwise, giving the simple saw-tooth patterns depicted in Fig. 1. This easily leads to the expression in Lemma 2 for the cost per time unit for holding returned products. In the remainder of this proof, we derive the expression in Lemma 2 for the final cost component; the serviceable inventory holding cost per time unit.

Fig. 2 depicts the ideal, i.e. with minimum cost, inventory pattern for each of the three classes defined in Section 3. The inventory levels and lengths of parts of the cycle that are indicated in the pictures can easily be checked. Note that two patterns $(\mathrm{a}, \mathrm{b})$ are presented for classes 2 and 3, depending on whether or not the production rates are such that the manufacturing and remanufacturing intervals overlap. The distinction is made for completeness only, as we will show that it does not affect the cost. We remark that since the patterns repeat themselves, it is not relevant to decide where the cycles begin/end. For presentational ease only, we start cycles when the inventory is zero.

Note that Class 1 is the only one where the stock drops to zero when either manufacturing or remanufacturing start, because for this case the production speed of either line is larger than the demand rate. For all classes, however, both manufacturing and remanufacturing start just in time and any delay would lead to a shortage.

Using Fig. 2, it can easily be checked that the total serviceable inventory cost for each class as given in Lemma 2 is correct.

\section{Appendix B. Proof of Lemma 3 (additional cost per time unit)}

Recall from Appendix A that the inventory for returned products follows a simple saw-tooth pattern, as is graphically depicted for an example in Fig. 1. Note also that this pattern is not influenced by the timing of manufacturing and remanufacturing batches. The timing of a remanufacturing batch for a product will obviously shift (in time) the saw-tooth to the left/right, but that does not affect the pattern or associated cost per time unit over a cycle.

The inventory pattern of serviceable products is more complex. That pattern and the associated cost per time unit do depend on the timing of (re)manufacturing batches. In Appendix A, we depicted the ideal timing and resulting patterns for three cases and derived the associated minimum costs per time unit of holding serviceable products (for a given cycle time). In this appendix, we will derive the additional costs for each case if the timing is not ideal.

Class 1

As the pattern for this class in Fig. 2 shows, the minimum cost is attained if remanufacturing starts $\left(1-\beta_{i}\right) T$ time units after manufacturing starts (and manufacturing starts $\beta_{i} T$ time units after remanufacturing starts). If this does not hold, then there are two possible cases:

(i) remanufacturing starts less then $\left(1-\beta_{i}\right) T$ time units after manufacturing starts,

(ii) remanufacturing starts more then $\left(1-\beta_{i}\right) T$ time units after manufacturing starts.

We first analyze case (i). This case occurs if the length of the time interval between successive manufacturing and remanufacturing starts is less than $T\left(1-\beta_{i}\right)$. Using the definition of $f(\cdot)$ in Section 2 , this length can be written as $f\left(x_{i}^{r}-x_{i}^{m}\right)$. To derive the additional serviceable holding costs as a result of starting remanufacturing 


\section{Class 1}

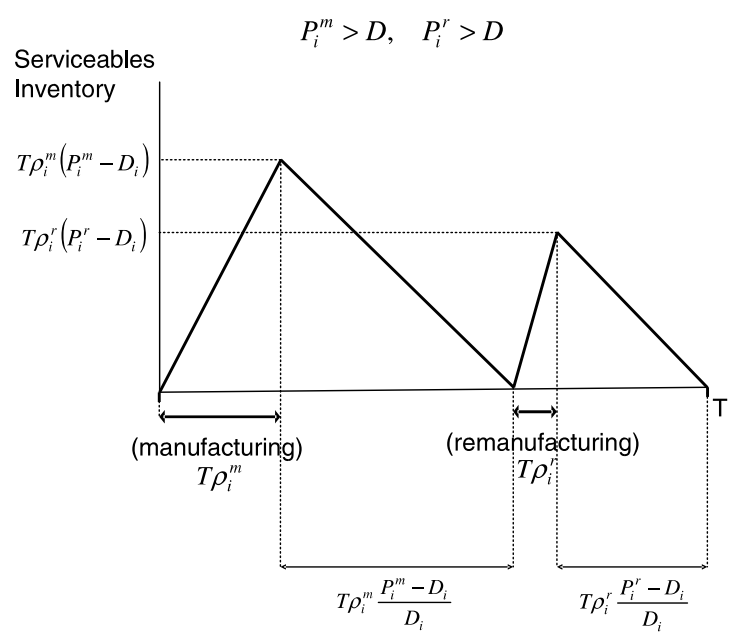

\section{Class 2a}

$P_{i}^{m}>D, \quad P_{i}^{r} \leq D$

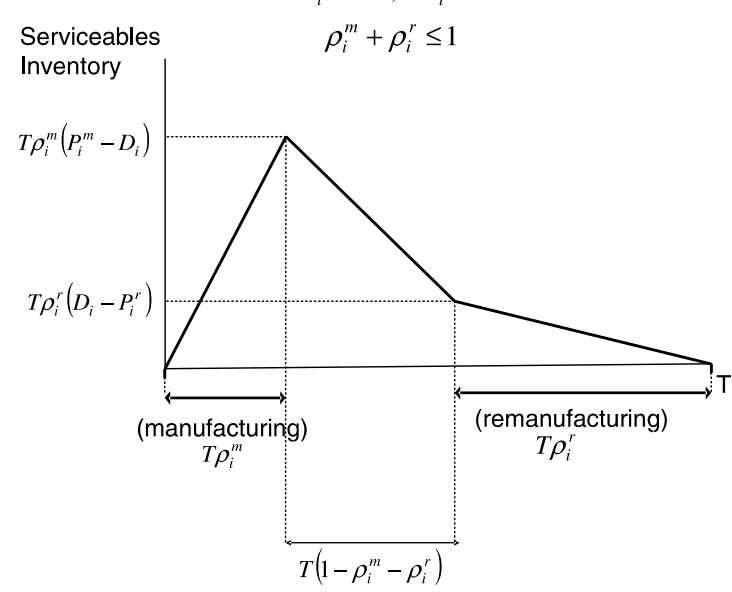

Class 3a

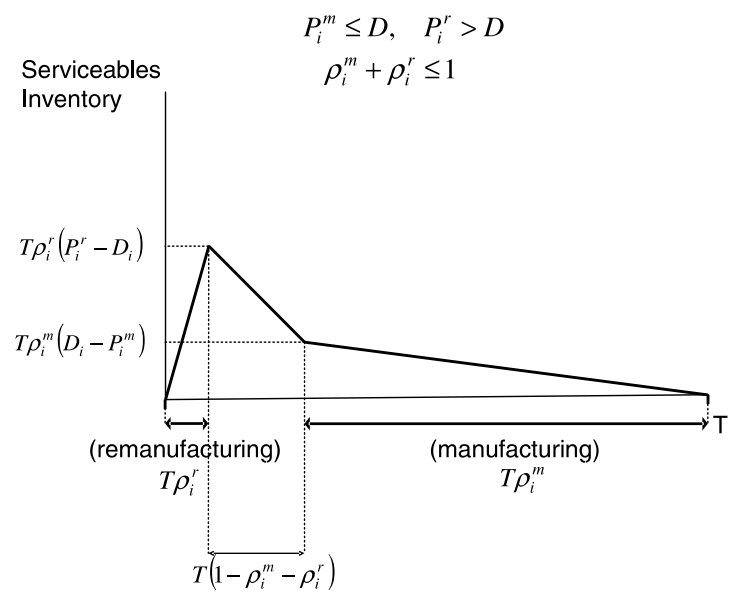

Class 2b

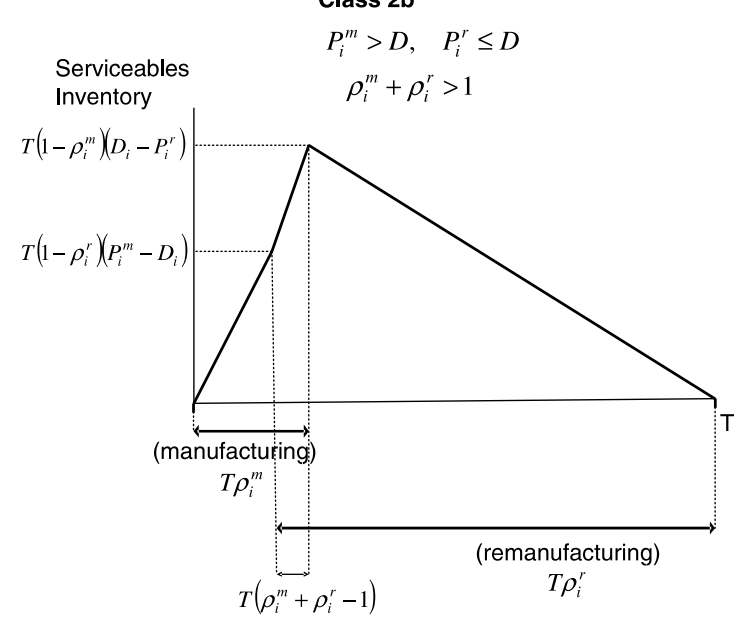

Class 3b

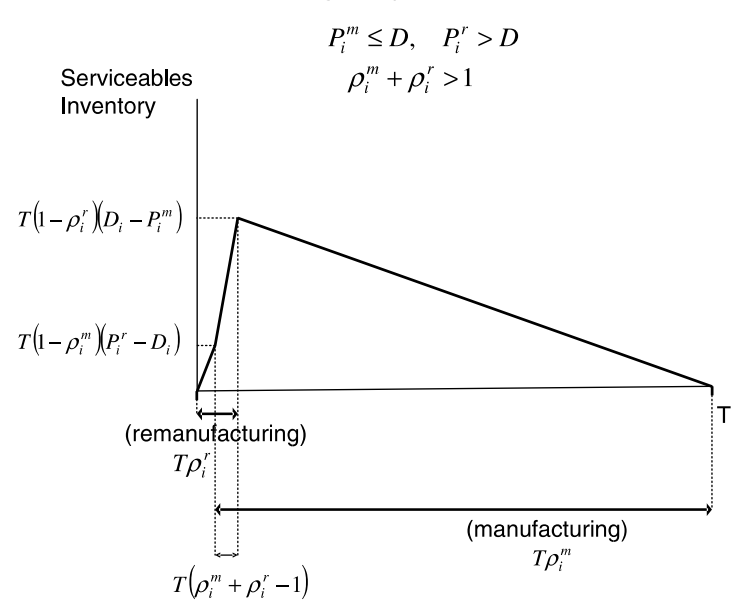

Fig. 2. Ideal serviceable inventory patterns for the three classes, with a subdivision for Classes 2 and 3 into situations where (a) $\rho_{i}^{m}+\rho_{i}^{r} \leqslant 1$ or (b) $\rho_{i}^{m}+\rho_{i}^{r}>1$. 
'too soon', we use a marginal cost argument. From the situation that remanufacturing takes place in time interval $\left[t, t+T \rho_{i}^{r}\right]$, suppose that it is shifted forward to $\left[t-\Delta, t+T \rho_{i}^{r}-\Delta\right]$. The effects are that (i) additional stock builds up at rate $P_{i}^{r}$ during $[t-\Delta, t]$, (ii) additional stock then remains constant at $\Delta P_{i}^{r}$ during $\left[t, t+T \rho_{i}^{r}-\Delta\right]$, and (iii) additional stock diminishes at rate $P_{i}^{r}$ during $\left[t+T \rho_{i}^{r}-\Delta, t+T \rho_{i}^{r}\right]$. This is illustrated in Fig. 3.

The additional total costs for shifting remanufacturing $\Delta$ time units forward are therefore $h_{i}^{s}\left(\Delta \frac{1}{2} \Delta P_{i}^{r}+\left(T \rho_{i}^{r}-\Delta\right) \Delta P_{i}^{r}+\Delta \frac{1}{2} \Delta P_{i}^{r}\right)=h_{i}^{s} T P_{i}^{r} \rho_{i}^{r} \Delta$ per cycle. Hence, the additional average costs for shifting remanufacturing $\Delta$ time units forward are $h_{i}^{s} P_{i}^{r} \rho_{i}^{r} \Delta$. Since, for any values of $x_{i}^{r}$ and $x_{i}^{m}$, the number of time units by which remanufacturing is shifted forward can be expressed as $\left[f\left(x_{i}^{r}-x_{i}^{m}\right)-T\left(1-\beta_{i}\right)\right]^{-}$, the additional cost is $h_{i}^{s} P_{i}^{r} \rho_{i}^{r}\left[f\left(x_{i}^{r}-x_{i}^{m}\right)-T\left(1-\beta_{i}\right)\right]^{-}$.

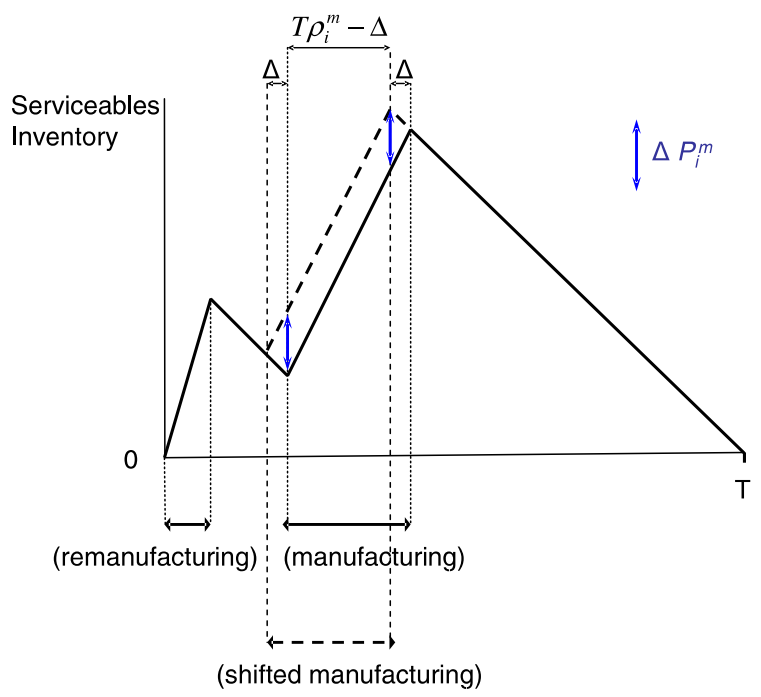

Fig. 3. The effect on the serviceable inventory of starting remanufacturing $\Delta$ time units earlier for Class 1 products.

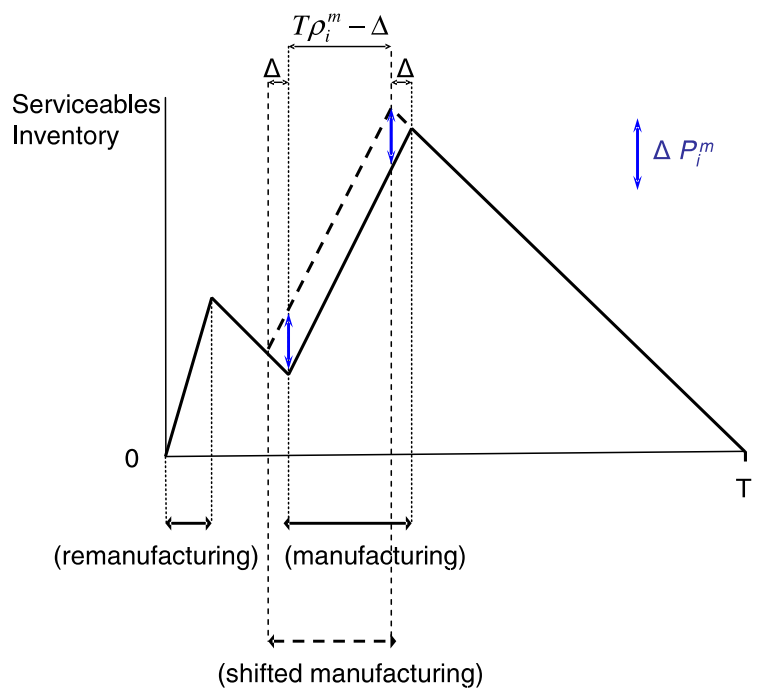

Fig. 4. The effect on the serviceable inventory of starting remanufacturing $\Delta$ time units later, or equivalently starting manufacturing earlier, for Class 1 products. 


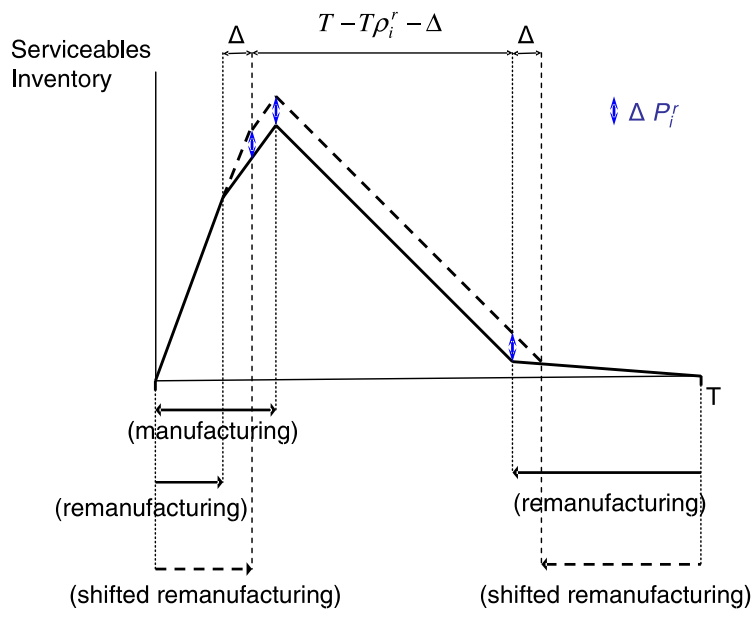

Fig. 5. The effect of starting remanufacturing $\Delta$ time units later for Class 2 products.

The analysis of the second case (ii) is similar. It is illustrated in Fig. 4 and leads to additional average costs of $h_{i}^{s} P_{i}^{m} \rho_{i}^{m}\left[f\left(x_{i}^{r}-x_{i}^{m}\right)-T\left(1-\beta_{i}\right)\right]^{+}$. Note from Fig. 4 that inventory now reaches its minimum (always set to zero, of course) when remanufacturing starts. So, in fact, it is more appropriate to think of a late remanufacturing start as an early manufacturing start. Although this just seems a matter of wording now, the insight will turn out to be useful in discussing the other classes.

Combining the results for the two cases, it follows that the expression given in Lemma 3 is correct for class 1.

Class 2

As can be seen from the ideal inventory patterns for these classes depicted in Appendix A, the minimum cost is attained if remanufacturing starts $\left(1-\rho_{i}^{r}\right) T$ time units after manufacturing starts. Using similar arguments as for Class 1, it can easily be checked that the additional costs for starting remanufacturing earlier are the same as for Class 1 .

However, the additional for starting remanufacturing more than $\left(1-\rho_{i}^{r}\right) T$ time units after manufacturing are different. Recall that for Class 1 products, inventory reaches its minimum when remanufacturing starts for this case (see also Fig. 2). For Class 2 products, inventory instead always reaches its minimum when manufacturing starts. As a result, the late remanufacturing start can no longer be interpreted as an early manufacturing start, and this affects the additional costs. Using Fig. 5, we get additional average costs of $h_{i}^{s} P_{i}^{r}\left(1-\rho_{i}^{r}\right)$ per time unit.

Class 3

The analysis is similar to that for class 2 .

Therefore, Lemma 3 holds for all classes of products.

\section{References}

Axsäter, S., 2006. Inventory Control. Springer Science+Business Media, New York, p. 177.

Bollapragada, R., Rao, U., 1999. Single-stage resource allocation and economic lot scheduling on multiple, nonidentical production lines. Management Science 45 (6), 889-904.

Elmaghraby, S.E., 1978. The economic lot scheduling problem (ELSP): Review and extensions. Management Science 24 (6), $587-598$.

Guide, V.D.R., 2000. Production planning and control for remanufacturing: Industry practice and research needs. Journal of Operations Management 18 (4), 467-483.

Sundin, E., Tang, O., Marten, E., 2005. The Swedish remanufacturing industry: An overview of present status and future potential. Paper presented at the 12th CIRP Life Cycle Engineering Seminar, Grenoble, France, April 3-5, 2005.

Tang, O., Teunter, R.H., 2006. Economic lot scheduling problem with returns. Production and Operations Management 15 (4), $488-497$.

Winston, W.L., 1991. Operations Research Applications and Algorithms, second ed. PWS-Kent Publishing Company, Boston, pp. 460464. 